\title{
Adaptive Channel Estimation Techniques for MIMO OFDM Systems
}

\author{
Md. Masud Rana \\ Dept. of Electronics and Radio Engineering \\ Kyung Hee University \\ South Korea \\ mrana928@yahoo.com
}

\author{
Md. Kamal Hosain \\ School of Engineering \\ Deakin University \\ Victoria-3217, Australia
}

\begin{abstract}
In this paper, normalized least mean (NLMS) square and recursive least squares (RLS) adaptive channel estimator are described for multiple input multiple output (MIMO) orthogonal frequency division multiplexing (OFDM) systems. These CE methods uses adaptive estimator which are able to update parameters of the estimator continuously, so that the knowledge of channel and noise statistics are not necessary. This NLMS/RLS $\mathrm{CE}$ algorithm requires knowledge of the received signal only. Simulation results demonstrated that the RLS CE method has better performances compared NLMS CE method for MIMO OFDM systems. In addition, the utilizing of more multiple antennas at the transmitter and/or receiver provides a much higher performance compared with fewer antennas. Furthermore, the RLS CE algorithm provides faster convergence rate compared to NLMS CE method. Therefore, in order to combat the more channel dynamics, the RLS CE algorithm is better to use for MIMO OFDM systems.
\end{abstract}

Keywords- MIMO; NLMS; OFDM; RLS

\section{INTRODUCTION}

Recently, multiple input multiple output (MIMO) channels have been introduced to achieve high data speed requisite by the next-generation communication systems [1]. The use of MIMO channels provides higher spectral efficiency versus single input single output (SISO), single input multiple output (SIMO), and multiple input single-output (MISO) channels, when the available bandwidth is inadequate. Furthermore, the diversity gain of the MIMO channels is nearly of second order when channel matrix has full rank. Consequently, by employing MIMO channels, not only the mobility of the wireless communications can be increased but also the algorithm can be more robust against fading, which makes it efficient for the requirements of the next-generation wireless services such as wireless local area networks (WLANs), worldwide interoperability for microwave access (WiMAX), wireless fidelity (WiFi), cognitive radio, and 3rd generation partnership project (3GPP) long term evolution (LTE) [2].

In SISO flat channels, channel estimation (CE) and its precision do not have a drastic impact on the performance of the receiver. Whereas in outdoor MIMO channels, the precision and speed of convergence of the channel estimator can drastically affect the performance of the receiver [3]. In SISO communications, the channel estimators may or may not use the training sequence or not. Although the distribution of the training symbols in a block of data affects the performance of systems [4], but due to simplicity, it is conventional to use the training symbols in the first part of each block. If the training sequence is not used, the estimator is called the blind channel estimator. A blind channel estimator uses information latent in statistical properties of the transmitting data [5]. In full-rank MIMO channels, the use of an initial training data is mandatory, and without it, the channel estimator does not converge [2], [5].

Orthogonal frequency division multiplexing (OFDM) systems have attracted much attention as a promising technology in wireless communication systems. In OFDM systems, the whole spectrum is divided into several subcarriers, and before each OFDM block the cyclic prefix (CP) is inserted. So, OFDM systems can mitigate the effects of multipath and have high spectrum efficiency. Therefore, OFDM is the important technique for next-generation communication. However, it is strict about $\mathrm{CE}$ to exploit the coherent demodulation, detection and decoding [7].

Several CE techniques have been proposed to mitigate interchannel interference (ICI) in OFDM systems. In [8], the least square (LS) CE has been proposed to minimize the squared differences between the received and estimated signal. The LS algorithm, which is independent of the channel model, is commonly used in equalization and filtering applications. But the statistics of channels in real world change over time and inversion of the large dimensional square matrix turns out to be ill-conditioned. To further improve the accuracy of the estimator, Wiener filtering based iterative $\mathrm{CE}$ has been investigated [9], [10]. However, this scheme also requires high complexity and knowledge of channel correlations.

The most important research topic in the wireless communications is the adaptive CE where the channel is rapidly time-varying. The time-varying multipath channel can be represented by a tap-delayed line with time varying coefficients and fixed tap spacing. An adaptive algorithm is a process that changes its parameters as it gain more information of its possibly changing environment. Among numerous iterative techniques that exist in the open literature, the popular category of approaches which are obtain from the minimization 
of the mean square error (MSE) between the output of the filter and desired signal to perform CE [10-15].

In this paper, normalized least mean (NLMS) square and recursive least squares (RLS) adaptive channel estimator are described for MIMO OFDM systems. These CE methods uses adaptive estimator which are able to update parameters of the estimator continuously, so that knowledge of channel and noise statistics are not required. This NLMS/RLS CE algorithm requires knowledge of the received signal only. This can be done in a digital communication system by periodically transmitting a training sequence that is known to the receiver. Simulation results show that the RLS CE method has better performances compared NLMS CE method for MIMO OFDM systems. In addition, the utilizing of more multiple antennas at the transmitter and/or receiver provides a much higher performance compared with fewer antennas. Furthermore, the RLS CE algorithm provides faster convergence rate compared to NLMS CE method. Therefore, in order to combat the channel dynamics, the RLS CE algorithm is better to use for MIMO OFDM systems.

We use the following notations throughout this paper: bold face and upper lower letter are used to represent matrix and vector. Superscripts $\mathrm{x}^{*}$ and $\mathrm{x}^{\mathrm{T}}$ denote the conjugate and conjugate transpose of the complex vector $\mathrm{x}$ respectively, and the symbol $\mathrm{E}($.$) denotes expectation.$

The remainder of the paper is organized as follows. The NLMS/RLS CE scheme is presented in section II, and its performance is analyzed in section III. Finally, some concluding remarks are given in section IV.

\section{CE METHODS}

\section{A. LMS CE Method}

An adaptive algorithm is a process that changes its parameters as it gain more information of its possibly changing environment. Among numerous iterative techniques that exist in the open literature, the popular category of approaches which are obtain from the minimization of the MSE between the output of the filter and desired signal to perform CE as shown in Fig. 1.

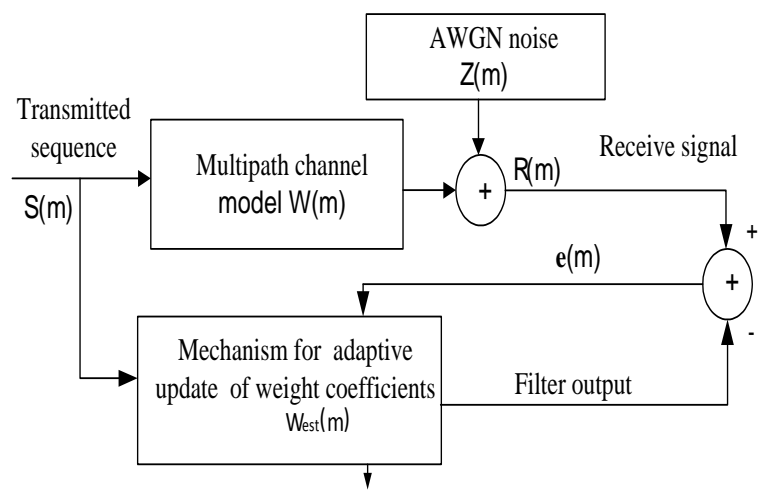

Figure 1. Scheme for adaptive CE.

The signal $\mathrm{S}(\mathrm{m})$ is transmitted via a time-varying channel $\mathrm{W}(\mathrm{m})$, and corrupted by an additive noise estimated by using any kind of CE method. The main aim of most channel estimation algorithms is to minimize the mean squared error (MMSE) i.e., between the received signal and its estimate [1620]. In the Fig 1, we have unknown multipath fading channel, that has to be estimated with an adaptive filter whose weight are updated based on some criterion so that coefficients of adaptive filter should be as close as possible to the unknown channel. The output from the channel can be expressed as:

$$
R(m)=\sum_{l=0}^{L-1} W(m, l) S(m-l)+Z(m),
$$

where $\mathrm{S}(\mathrm{m}-\mathrm{l})$ is the complex symbol drawn from a constellation $s$ of the lth paths at time $\mathrm{m}-\mathrm{l}, \mathrm{L}$ is the channel length, $\mathrm{Z}(\mathrm{m})$ is the AWGN with zero mean and variance $\sigma^{2}$. The above equation can be rewritten as vector notation [1]:

$$
\begin{aligned}
& R(m)=W(m) S(m)+Z(m), \\
& \quad \text { The output of the adaptive filter is } \\
& Y(m)=W_{\text {est }}(m) S(m),
\end{aligned}
$$

where $W_{e s t}(m)$ is the estimated channel coefficients at time $\mathrm{m}$. The priori estimated error signal needed to update the weights of the adaptive filter is

$$
\begin{aligned}
e(m) & =R(m)-Y(m) \\
& =W(m) S(m)+Z(m)-W_{\text {est }}(m) S(m)
\end{aligned}
$$

This error signal is used by the $\mathrm{CE}$ to adaptively adjust the weight vector so that the MSE is minimized. Now the cost function $j(m)=E\left[e(m) e^{*}(m)\right]$ for the adaptive filter structure is

$$
\begin{aligned}
j(m)= & E[R(m) R(m)]-E[S] R^{*} W_{e s t}(m)-R(m) W_{e s t}(m) \\
& E[S]-W_{e s t}(m) W_{e s t}(m) E[S(m) S(m)] S \\
= & \sigma_{r}{ }^{2}-C(m) W_{e s t}(m)-W_{e s t}(m) C(m) \\
& +D(m) W_{e s t}^{T}(m) W_{e s t}(m)
\end{aligned}
$$

where $\sigma_{r}{ }^{2}$ is the variance of the received signal, $C(m)=E[S(m) R(m)]$ is the cross-correlation vector between the tap input vector $S(m)$ and the received signal $\mathrm{r}(\mathrm{m})$, and $D(m)=E\left[S(m) S^{T}(m)\right]$ is the correlation matrix of the tap input signal $\mathrm{S}(\mathrm{m})$. Now taking the gradient vector with respect to $W_{e s t}(m)$ :

$$
\begin{aligned}
\Delta j(m) & =-2 C(m)+2 D(m) W_{e s t}(m) \\
& =-2 S(m) R^{*}(m)+2 S(m) S(m) W_{e s t}(m) .
\end{aligned}
$$

According to the method of steepest descent, if $W_{\text {est }}(\mathrm{m})$ is the tap-weight vector at the mth iteration then the following recursive equation may be used to update $W_{\text {est }}(\mathrm{m})$ : 


$$
\begin{aligned}
W_{\text {est }}(m+1) & =W_{\text {est }}(m)-1 / 2 \eta \Delta j(m) \\
& =W_{\text {est }}(m)+\eta S(m)\left[R^{*}(m)-W_{\text {est }}(m) S(m)\right] \\
& =W_{\text {est }}(m)+\eta S(m) e^{*}(m),
\end{aligned}
$$

where $\mathrm{W}_{\text {est }}(\mathrm{m}+1)$ denotes the weight vector to be computed at iteration $(m+1)$ and $\eta$ is the LMS step size which is related to the rate of convergence. The smaller step size means that a longer reference or training sequence is needed, which would reduce the payload and hence, the bandwidth available for transmitting data. The term $\left[\eta S(m) e^{*}(m)\right]$ represents the correction factor or adjustment that is applied to the current estimate of the tap-weight vector. In order to improved system performance, taking into account the variation in the signal level at the filter input and selects a normalized step size parameter i.e.,

$$
W_{\text {est }}(m+1)=W_{\text {est }}(m)+\frac{\eta S(m)}{S(m) S(m)} e^{*}(m)
$$

The iterative procedure is started with an initial guess $\mathrm{W}_{\text {est }}(0)$. Therefore, the NLMS based CE is least sensitive to the scaling of its input signal variation. Therefore, this algorithm is able to sense the best possible channel coefficients are changing.

\section{B. RLS CE Method}

The RLS CE requires all the past samples of the input and the desired output is available at each iteration. The objective function of a RLS CE algorithm is defined as an exponential weighted sum of errors squares:

$$
\mathrm{c}(\mathrm{m})=\sum_{\mathrm{m}=1}^{\mathrm{n}} \lambda^{\mathrm{n}-\mathrm{m}} \mathbf{e}^{\mathrm{H}}(\mathrm{m}) \mathbf{e}(\mathrm{m})+\delta \lambda^{\mathrm{n}} \mathrm{W}^{\mathrm{H}}(\mathrm{m}) \mathrm{W}(\mathrm{m}),
$$

where $\delta$ is a positive real number called regularization parameter, $\mathrm{e}(\mathrm{m})$ is the prior estimation error, and $\lambda$ is the exponential forgetting factor with $0<\lambda<1$. The prior estimation error is the difference between the desired response and estimation signal:

$$
\mathbf{e}(\mathrm{m})=\mathrm{H}(\mathrm{m})-\mathrm{W}^{\mathrm{H}}(\mathrm{m}) \mathrm{S}(\mathrm{m})
$$

The objective function is minimized by taking the partial derivatives with respect to $\mathrm{W}(\mathrm{n})$ and setting the results equal to zero.

$$
\begin{aligned}
& \frac{\delta C(m)}{\delta W(m)}=0=-2 \sum_{m=1}^{n} \lambda^{n-m} S(m) e^{H}(m)+2 \delta \lambda^{n} W(m) \\
& =-2 \sum_{m=1}^{n} \lambda^{n-m} S(m)\left[H(m)-W^{H}(m) S(m)\right]^{H}+2 \delta \lambda^{n} W(m) \\
& W(m)\left[\sum_{m=1}^{n} \lambda^{n-m} S(m) S^{H}(m)+\delta \lambda^{n} I\right]=\sum_{m=1}^{n} \lambda^{n-m} S(m) H^{H}(m) \\
& R_{s}(m) W(m)=R_{s h}(m) \\
& W(m)=R_{s}^{-1}(m) R_{s h}(m)
\end{aligned}
$$

where $\mathbf{R}_{\mathrm{s}}(\mathrm{m})$ is the transmitted auto-correlation matrix $R_{s}(m)=\sum_{m=1}^{n} \lambda^{n-m} S(m) S^{H}(m)+\delta \lambda^{n} I=\lambda R_{s}(m-1)+S(m) S^{H}(m)$ and $\quad \mathbf{R}_{\mathrm{sh}}(\mathrm{m})$ is the cross correlation matrix i.e., $R_{s h}(m)=\sum_{m=1}^{n} \lambda^{n-m} S(m) H^{H}(m)=\lambda R_{s h}(m-1)+S(m) H^{H}(m)$.

According to the Woodbury identity, the above $\mathbf{R}_{\mathrm{sh}}(\mathrm{m})$ can be written as

$$
\begin{gathered}
R_{s h}^{-1}(m)=\lambda^{-1} R_{s h}^{-1}(m-1)-\frac{\lambda^{-2} R_{s h}^{-1}(m-1) S(m) S^{H}(m) R_{s h}^{-1}(m-1)}{1+\lambda^{-1} S^{H}(m) R_{s h}^{-1}(m-1) S(m)} \\
\text { For }
\end{gathered}
$$

convenience of computing, let $\mathrm{D}(\mathrm{m})=\mathrm{R}_{\mathrm{sh}}(\mathrm{m})$ and

$$
K(m)=\frac{\lambda^{-1} D(m-1) S(m)}{1+\lambda^{-1} S^{H}(m) D(m-1) S(m)}
$$

The $K(m)$ is referred as a gain matrix. We may rewrite (9) as:

$$
D(m)=\lambda^{-1} D(m-1)-\lambda^{-1} K(m) s^{H}(m) D(m-1)
$$

So simply (13) to

$$
K(m)=D(m) S(m)=R_{s h}^{-1}(m) S(m)
$$

Substituting (14), (15) into (11), we obtain the following RLS CE formula

$$
\begin{aligned}
W(m)= & W(m-1)+K(m)\left[H(m)-W^{H}(m-1) s(m)\right]^{H} \\
= & W(m-1)+K(m) \varepsilon^{H}(m), \\
& \text { where } \varepsilon(\mathrm{m}) \text { is a prior estimation error as } \\
\varepsilon(m)= & H(m)-W^{H}(m-1) S(m)
\end{aligned}
$$

Therefore, equation (17) is the recursive RLS CE algorithm to update channel coefficient.

\section{ANALYTICAL RESUltS}

The error performance of the aforementioned iterative estimation algorithm is explored by performing extensive computer simulations. In these simulations, we consider 2 by 2 , 4 by 4,6 by 6 , and 8 by 8 MIMO OFDM systems. The data symbol is based on Q-PSK modulation. The forgetting factor is 0.9 and learning rate is 0.4 , and signal to noise ratio is $15 \mathrm{~dB}$. From the simulation results, one can observed that the RLS CE method has better performances compared NLMS CE method. In addition, the utilizing of more multiple antennas at the transmitter and/or receiver provides a much higher BER 
performance compared with fewer antennas. Furthermore, the RLS CE algorithm provides faster convergence rate compared to NLS CE method. Therefore, in order to combat the channel dynamics, the RLS CE algorithm is better to use for OFDM systems. But the RLS CE algorithm is suffered from a computational complexity point of view. In addition, the RLS algorithm has the recursive inversion of an estimate of the autocorrelation matrix of the input signal as its cornerstone; problems arise, if the autocorrelation matrix is rank deficient.

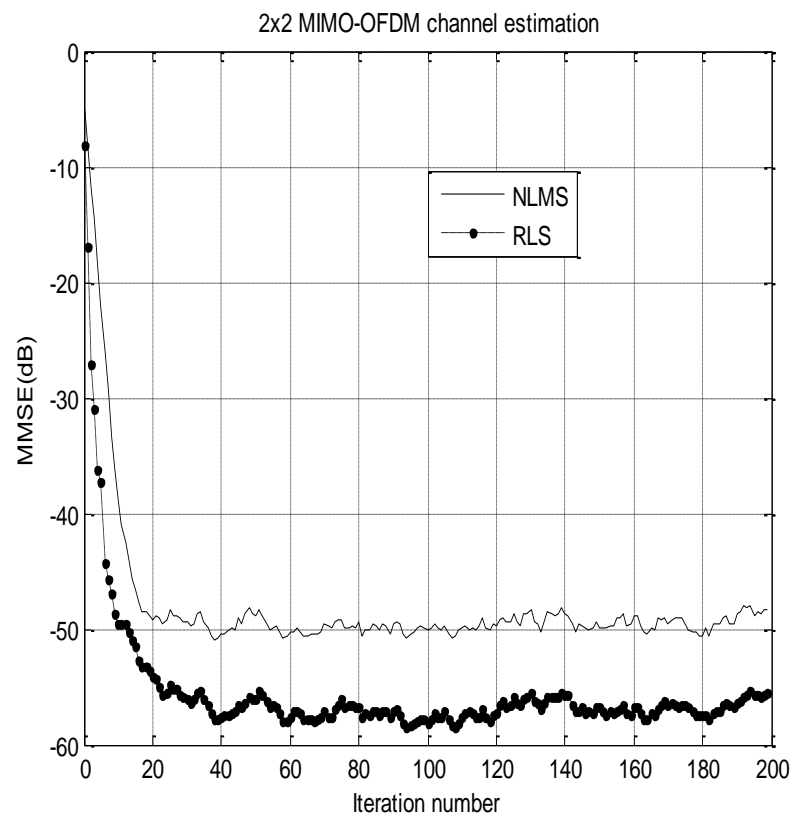

Figure 2. 2 by 2 MIMO systems

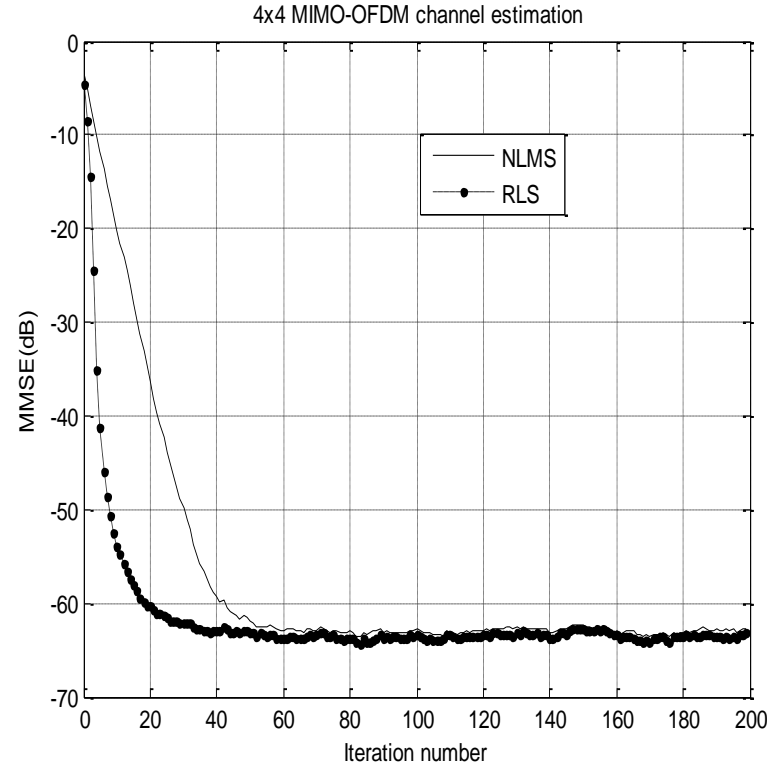

Figure 3. 4 by 4 MIMO systems

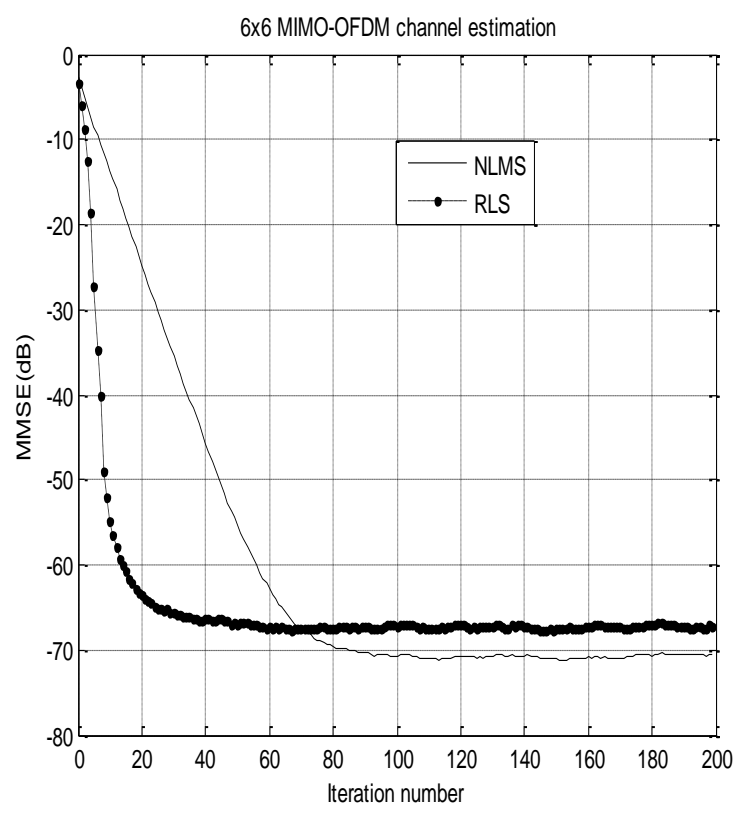

Figure 4.6 by 6 MIMO systems

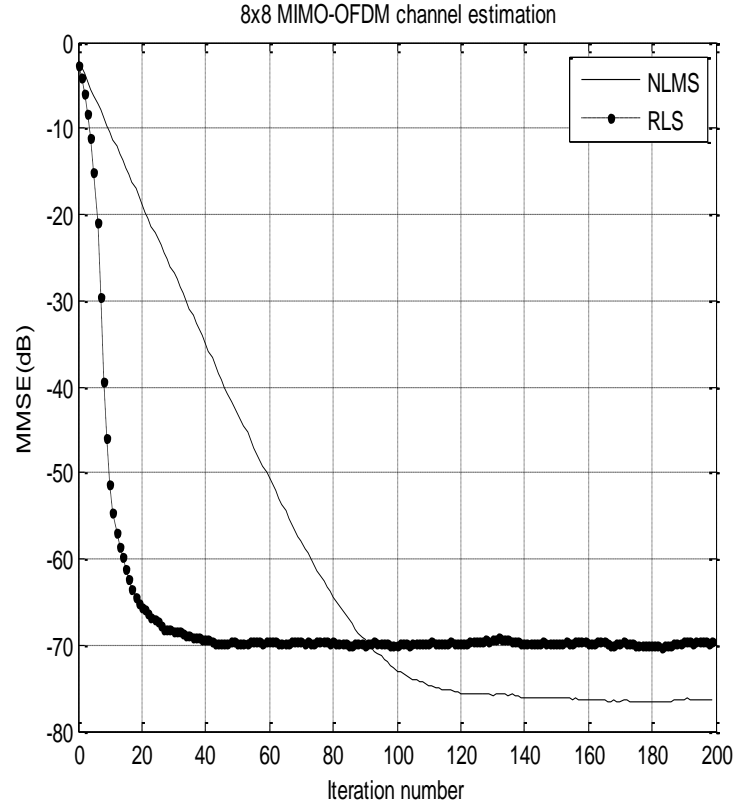

Figure 5.8 by 8 MIMO systems

\section{CONCLUSION}

Recently, multiple input multiple output (MIMO) transmission has been well known as one of the most important practical technique to combat fading as well as increase the channel capacity of wireless communication systems. In this paper, NLMS and RLS adaptive channel estimator are described for MIMO OFDM systems. Simulation results demonstrated that the RLS CE method has better performances compared NLMS CE method for MIMO OFDM systems. In addition, the utilizing of more multiple antennas at the transmitter and/or receiver provides a much higher 
performance compared with fewer antennas. Therefore, in order to combat the channel dynamics, the RLS CE algorithm is better to used for MIMO OFDM systems.

\section{REFERENCES}

[1] G. J. Foschini and M. J. Gans, "On limits of wireless communications in a fading environment when using multiple antennas," Wireless Pers. Commun., vol. 6, no. 3, pp. 311-335, Mar. 1998.

[2] E. Karami, "Tracking performance of least squares MIMO channel estimation algorithm," IEEE Trans. On Wireless Comm. vol. 55, no.11, pp. 2201-2209, Nov. 2007.

[3] V. Pohl, P. H. Nguyen, V. Jungnickel, and C. Von Helmolt, "How often channel estimation is needed in MIMO systems," in Proc. IEEE Global Telecommun. Conf., no. 1, pp. 814-818. Dec. 2003,

[4] S. Addireddy, L. Tong, and H. Viswanathan, "Optimal placement of training for frequency-selective block-fading channels," IEEE Trans. Inf. Theory, vol. 48, no. 8, pp. 2338-2353, Aug. 2002.

[5] L. Tong, "Blind sequence estimation," IEEE Trans. On Commun., vol. 43, no. 12, pp. 2986-2994, Dec. 1995.

[6] A. Vosoughi and A. Scaglione, "Channel estimation for precoded MIMO systems," in Proc. IEEE Workshop Statist. Signal Process., pp. 442-445, Sep. 28-Oct. 1, 2003.

[7] J. Li, J. Ma, S. Liu "RLS channel estimation with superimposed training," in Proc. Int. Con. on Comm. Technology, 2008.

[8] A. Ancora, C. B. Meili, and D. T. Slock, "Down-sampled impulse response least-squares channel estimation for LTE OFDMA," in Proc. Int. Con. on Acoustics, Speech, and Signal Processing, pp. III-293-III296, Apr. 2007.

[9] L. A. M. R. D. Temino, C. N. I Manchon, C. Rom, T. B. Sorensen, and P. Mogensen, "Iterative channel estimation with robust wiener filtering in LTE downlink," in Proc. Int. Con. on VTC, pp. 1-5, Sept. 2008.

[10] J. J. V. D. Beek, O. E. M. Sandell, S. K. Wilsony, and P. O. Baorjesson, "On channel estimation in OFDM systems," in Proc. Int. Con. on VTC, vol. 2, pp. 815-819, July 1995.

[11] S. Haykin, "Adaptive Filter Theory," Prentice-Hall International Inc, 1996.

[12] F. Adachi, H. Tomeba, and K. Takeda, "Frequency-domain equalization for broadband single-carrier multiple access," IEICE Trans. on Commun., vol. E92-B, no. 5, pp. 1441-1456, May 2009.

[13] S. Yameogo, J. Palicot, and L. Cariou, "Blind time domain equalization of SC-FDMA signal," in Proc. Vehicular Technology Conference, Sept. 2009, pp. 14.

[14] S. Y. Park, Y. G. Kim, and C. G. Kang, "Iterative receiver for joint detection and channel estimation in OFDM systems under mobile radio channels," IEEE Trans. on Vehicular Technology, vol. 53, no. 2, pp. 450-460, Mar. 2004.

[15] J. Berkmann, C. Carbonelli, F. Dietrich, C. Drewes, and W. Xu, "On 3G LTE terminal implementation-standard, algorithms, complexities and challenges," in Proc. Wireless Commun. and Mobile Computing Conference, Aug. 2008, pp. 970-975.

[16] W. C. Jakes, Ed., Microwave mobile communications. New York: Wiley-IEEE Press, Jan. 1994.

[17] B. Karakaya, H. Arslan, and H. A. Curpan, "Channel estimation for LTE uplink in high Doppler spread," in Proc. Wireless Commun. And Networking Conference, Apr. 2008, pp. 1126-1130.

[18] R. C. Alvarez, R. Parra-Michel, A. G. O. Lugo, and J. K. Tugnait, "Enhanced channel estimation using superimposed training based on universal basis expansion," IEEE Trans. on Signal Process., vol. 57, no. 3, pp. 1217-1222, Mar. 2009.

[19] A. Kalayciogle and H. G. Ilk, "A robust threshold for iterative channel estimation in OFDM systems," Radio Engineering journal, vol. 19, no. 1, pp. 32-38, Apr. 2010. 10

[20] Q. Li, G. Li, W. Lee, M. il Lee, D. Mazzarese, B. Clerckx, and Z. Li, "MIMO techniques in WiMAX and LTE: a feature overview," IEEE Commun. Magazine, vol. 48, no. 5, pp. 86-92, May. 2010. 